\title{
A new reconstruction algorithm for use with capacitance-based process tomography
}

\section{Ø. ISAKSEN†‡ and J. E. NORDTVEDT $\uparrow \S$}

Keywords: Process measurements, multiphase flow, tomography.

A new reconstruction algorithm for use with capacitance-based process tomography is proposed. A numerical simulator, capable of calculating the capacitances for a particular sensor configuration and flow regime is used together with a parameter representation of the dielectric distribution and an optimization algorithm. The algorithm calculates these parameters and hence the dielectric distribution, by minimizing a function defined as a weighted sum of square differences between the measured and estimated capacitances. The method is tested by using both synthetic and experimental data, and the results are compared with results from the commonly used Linear Back Projection (LBP) algorithm. The method is capable of obtaining the correct parameter values for all the flow regimes tested, and does provide a better estimate than the LBP method. The method proves to be very promising, and is a step towards quantitative capacitance tomography.

\section{Introduction}

Capacitance based tomography has been developed during the past decade, and is presently at the prototype level. The capacitance tomography system may be used for monitoring oil/gas distribution in pipes, and is capable of providing qualitative flow pattern information (Huang et al. 1989, Xie et al. 1991). The experimental set-up is shown in Fig. 1; a number of electrodes are mounted circumferentially around a pipe, and the capacitances between all combinations of electrode pairs are measured. The dielectric distribution within the pipe (e.g., the oil/gas flow pattern) is usually reconstructed using the Linear Back Projection (LBP) method (Xie et al. 1992).

Accurate quantitative information from such a system is, however, quite hard to obtain, due to the following three problems associated with the capacitance technique:

(1) No explicit relation exists between the dielectric distribution and the measured capacitances. (Note that there is an explicit relation, but it often requires a numerical solution.)

(2) The electric field between the source and detector electrodes determines the capacitance sensitivity distribution, which is non-uniform (higher near the electrodes and lower in the central region of the pipe).

(3) The number of measurements is small $(=N(N-1) / 2$, where $N$ is the number of electrodes). That is because there is a limit to how much the electrode size can be reduced due to the finite capacitance resolution of the sensor electronics.

Received 15 February 1993.

† University of Bergen, Department of Physics, Engineering Centre, Allégt. 55, N. 5007 Bergen, Norway.

$\ddagger$ Permanent affiliation: Christian Michelsen Research, Fantoftvg. 38, N-5036 Fantoft, Bergen, Norway.

$\S$ Permanent affiliation: Rogaland Research, P.O. Box 2503 Ullandhaug, N-4004 Stavanger, Norway.

A preliminary version of this paper was presented at the International Conference on Electronic Measurement and Instruments, Beijing, 20-22 October 1992. 


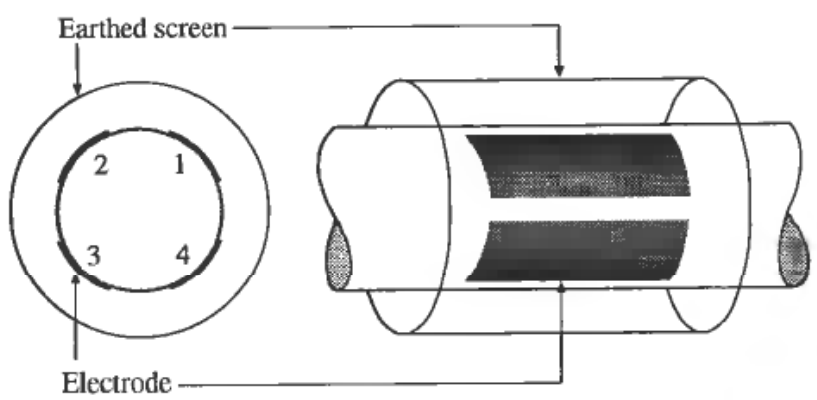

Figure 1. Sketch of the sensor configuration for a four-electrode capacitance tomography system.

Thus, the well-developed reconstruction methods for X-ray tomography cannot easily be adapted into a capacitance tomography system and consequently, the LBP method has traditionally been used for solving the inverse problem. Although the problem of non-uniform sensitivity may be accounted for by modifying the LBP algorithm, the method is in itself a 'smoothing' algorithm and the number of measurements is very limited (28 for an eight-electrode system). Hence, smoothing of sharp transitions in the dielectric constant, e.g. in the oil/gas distribution, will always occur. The LBP method is thus not strictly suitable for obtaining quantitative results.

To overcome the above mentioned problems, optimization theory has been tried in developing a new reconstruction algorithm (Isaksen and Nordtvedt 1992). In the present method, a numerical simulator, capable of calculating the capacitances for a particular sensor configuration and flow regimes, is used together with a parameter representation of the dielectric distribution and an optimization algorithm. The algorithm calculates these parameters, and thus obtains the dielectric distribution (e.g., the oil/gas flow pattern).

\section{Reconstruction}

In order to obtain flow pattern information, the measured capacitance values have to be related to the dielectric distribution within the pipe, i.e., the dielectric distribution has to be reconstructed from the measured data. This inverse problem can be solved in many different ways. However, due to the nature of the capacitance tomography system described above, the LBP algorithm is the most commonly used. In the simplest form of the LBP algorithm, the pipe is divided into a number of elements (or pixels) and the 'grey level' for each pixel is given by:

$$
g_{k}=\frac{1}{n} \cdot \sum_{i=1}^{n} C_{i}^{\text {norm }}
$$

where $C^{\text {norm }}$ is the normalized capacitance, $k$ is the pixel index, and $n$ is the number of measurements for which pixel $k$ is a part of the corresponding sensitivity area. The method may be improved by using sensitivity information provided by a numerical simulator. In this work, an improved LBP algorithm similar to the one developed by Xie $e t$ al. (1992) has been implemented, in order to be able to compare the results from the proposed algorithm with a state-of-the-art LBP procedure. The area inside the pipe is divided into 900 pixels, and by using 28 measurements (for an eight-electrode system) and 6 (for a four-electrode system) the LPB algorithm calculates the 'grey level' in each pixel. 


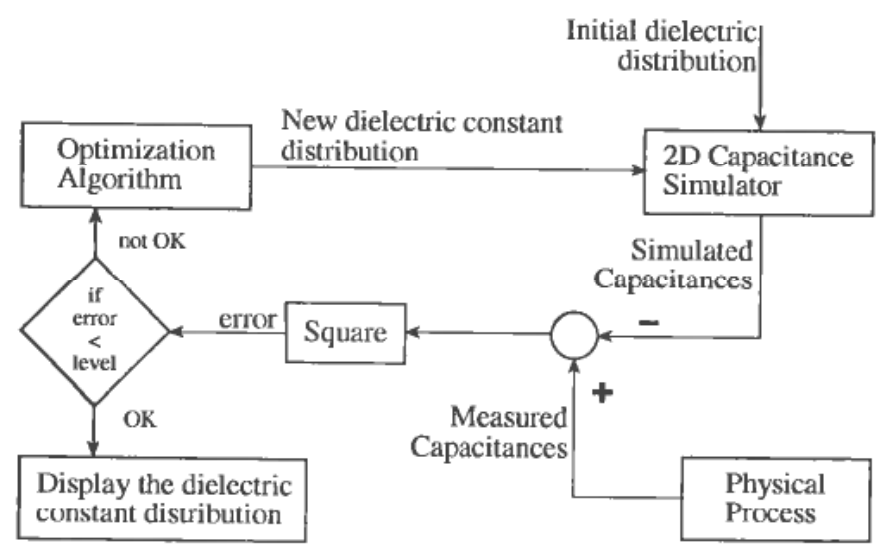

Figure 2. The implicit estimation procedure.

Alternatively, the dielectric distribution may be calculated using the implicit procedure shown in Fig. 2. The physical process is a multi-electrode capacitance tomography system, in which the potentials at the electrodes are altered in sequence in order to measure all the different capacitances possible (Isaksen 1989). Thus, the experimental outputs are 28 capacitances for an eight-electrode system. A mathematical simulator, capable of calculating the same capacitances for a given flow regime, defines the calculated outputs. The flow regime is defined by a set of parameters, and the parameters are modified in order to obtain the least possible discrepancy between the measured and calculated capacitances.

In order to be able to use an optimization procedure to calculate the dielectric distribution inside the pipe (i.e., calculate the flow regime), the flow regime has to be represented by a set of parameters (Isaksen and Nordtvedt 1992). Figure 3 shows the parameterization used in this work for representing the different flow regimes.

An optimization procedure is, in principle, an algorithm capable of minimizing or maximizing a function defined by a set of independent variables, or parameters. Hence, in order to be able to use an optimization algorithm to solve the present problem, a function has to be defined by the parameters describing the flow regime, the simulated capacitances, and the measurements. Such a function, most commonly denoted as an object function, is in this work defined by the equation:

$$
J(\boldsymbol{\beta})=\frac{1}{2} \sum_{i=1}^{M}\left(\frac{\boldsymbol{F}_{i}^{\text {meas }}-\boldsymbol{F}_{i}^{\text {est }}(\boldsymbol{\beta})}{\boldsymbol{F}_{i}^{\text {meas }}}\right)^{2}
$$

where $M$ is the number of measurements, $\left\{\boldsymbol{F}^{\text {meas }}\right\}_{i=1}^{M}$ is the measured capacitances, $\left\{\boldsymbol{F}^{\mathrm{est}}(\boldsymbol{\beta})\right\}_{i=1}^{M}$ is the capacitance calculated by the mathematical simulator, and $\boldsymbol{\beta}$ is the parameters describing the different flow regimes. For the parameterization shown in Fig. 3, the $\beta$ equals $\left[x_{1}, y_{1}, r_{1}, x_{2}, y_{2}, r_{2}\right]^{\mathrm{T}},\left[x, y, a, b, \varepsilon_{\text {mix }}\right]^{\mathrm{T}}$, and $[\theta, d]^{\mathrm{T}}$ for Fig. $3(a),(b)$ and $(c)$ respectively. Several methods exist for minimizing Eqn. 2. However, we have initially used the Gauss-Newton Levenberg-Marquardt (Gill et al. 1981). In this method the step towards a minimum for Eqn. 2 is a function of the first order partial derivatives of the object function. The method has been used with success in other applications (Watson et al. 1988, Aga 1991, Nordtvedt et al. 1991), and proven to be rather robust procedure.

As can be seen from Fig. 2, the new reconstruction algorithm uses a numerical model of the capacitance sensor system. A 2D capacitance simulator, based on a finite 


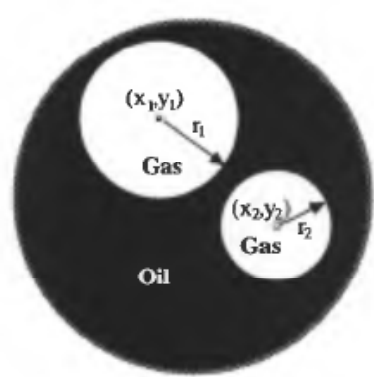

(a)

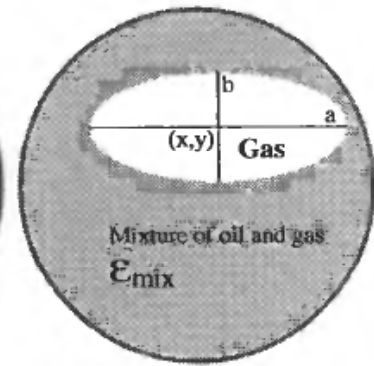

(b)

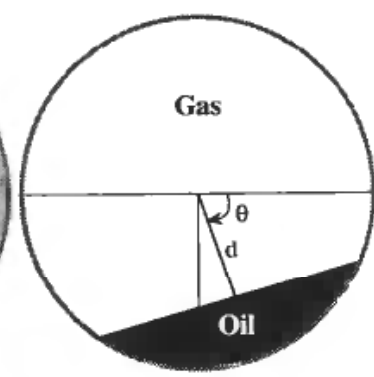

(c)

Figure 3. The different parameterization used in this work. (a) Two gas bubbles in the oil; one located at $\left(x_{1}, y_{1}\right)$ with radius $r_{1}$, and one at $\left(x_{2}, y_{2}\right)$ with radius $r_{2}$. (b) An elliptically shaped gas bubble located at $(x, y)$ with form parameters $a$ and $b$. The oil continuous phase consists of a mixture of oil and gas with a dielectric constant given by $\varepsilon_{\text {mix }}(c)$ Stratified flow characterized by $\theta$ and $d$.

element solution of the Poisson's equation, has been developed (Isaksen 1989). It is capable of calculating all the capacitances in a multi-electrode sensor system for a given flow regime. This simulator has been verified by use of experimental data and a 3D capacitance simulator. The overall accuracy is better than $2 \%$ (Isaksen and Nordtvedt 1992).

\section{Results and discussion}

Various flow regimes have been reconstructed by using synthetic and experimental data from a four- and an eight-electrode system. Both the modified LBP and the new reconstruction algorithms have been tested. The results shown for the eight-electrode system are based on synthetic data (i.e., data generated by the simulator), and the results shown from the four-electrode system are based on experimental measurements on static flow regimes.

\subsection{Results based on synthetic data}

The results are based on an eight-electrode system similar to the system shown in Fig. 1, using the following sensor construction parameters: $41 \mathrm{~mm}$ inner pipe radius, $4 \mathrm{~mm}$ pipe wall made of perspex, screen radius at $60 \mathrm{~mm}$, and eight electrodes mounted circumferentially around the pipe (the angle between two adjacent electrodes is $10^{\circ}$ ). To generate the data a mathematical simulator has been used. Hence, the estimation errors due to inadequate modeling and inaccurate measurement do not occur, because the 'measurement' and the 'estimates' are produced by using the same simulator.

Figure 4 shows the reconstruction of a large gas bubble in a gas/oil flow in a pipe. In addition, small gas bubbles are also present in the oil phase. The pure oil and the oil/bubble mixture are assumed to have a dielectric constant of 2.167 and 1.6 respectively. Figure $4(c)$ shows the flow regime reconstructed by the improved LPB. It can clearly be seen that there is a circular area of high gas concentration in the first quadrant: however, it is hard to tell where the phase interface is located. Further, the oil continuous phase consists of an oil/gas mixture, and not pure oil. The reconstruction with the new algorithm (see Fig. 4(d)) produces an image which is identical to the true flow regime. Figure $4(b)$ shows how the parameters $\left(x, y, r\right.$ and $\left.\varepsilon_{\text {mix }}\right)$ are converging toward the solutions as a function of a number of iterations. The initial dielectric distribution is given by $x=-10 \mathrm{~mm}, y=15 \mathrm{~mm}, r=10 \mathrm{~mm}$ and $\varepsilon_{\text {mix }}=5 \cdot 0$. Figure $4(e)$ shows the square root of the object function plotted as a function of iterations for 


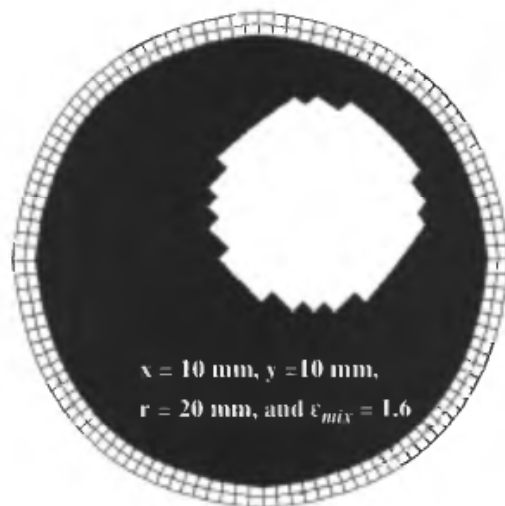

(a)

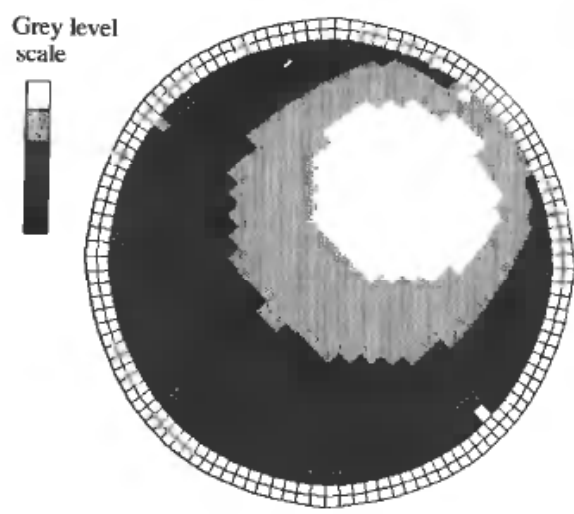

(c)

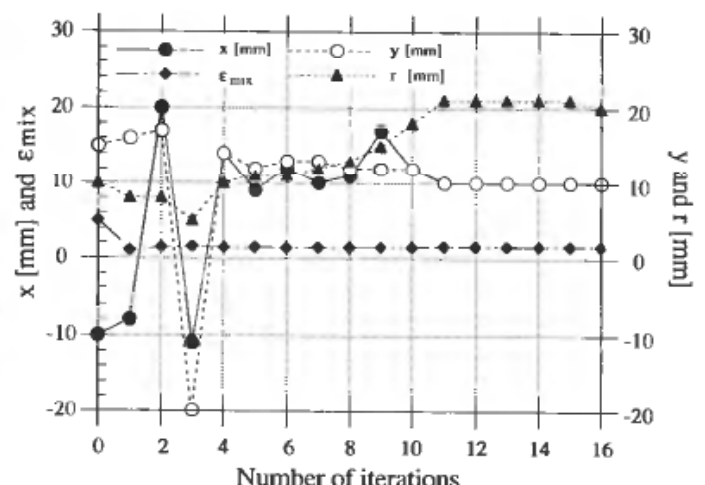

(b)

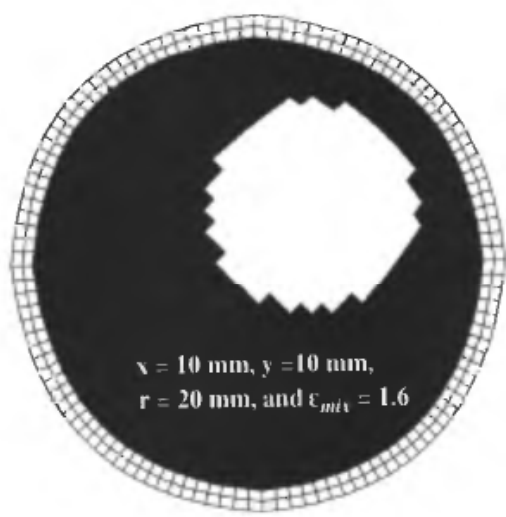

(d)

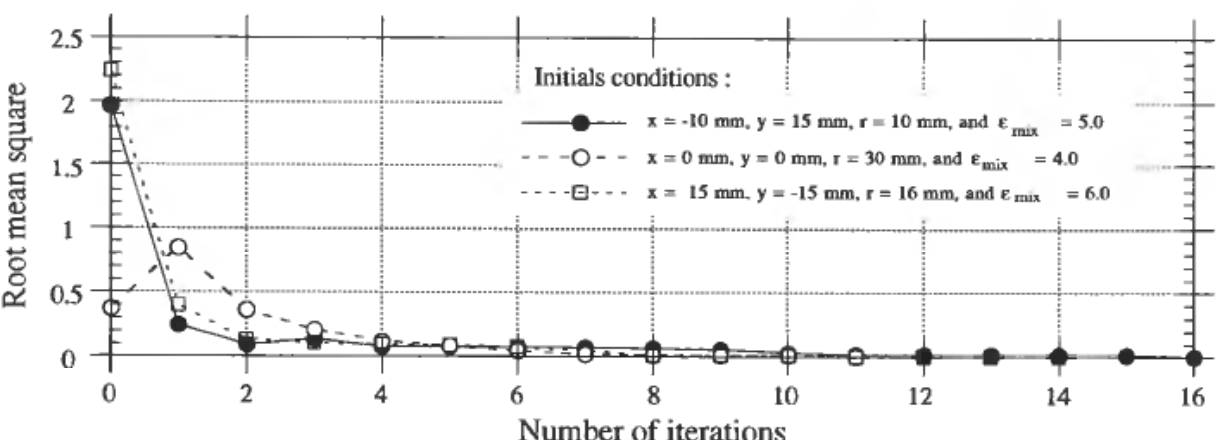

(e)

Figure 4. Reconstruction of a large gas bubble defined by $x=10 \mathrm{~mm}, y=10 \mathrm{~mm}, r=20 \mathrm{~mm}$, and $\varepsilon_{\operatorname{mix}}=1.6:(a)$ The true oil/gas distribution. $(b) x, y, r$, and $\varepsilon_{\text {mix }}$ as a function of the number of iterations. The initial dielectric distribution was: $x=-10 \mathrm{~mm}, y=15 \mathrm{~mm}$, $r=10 \mathrm{~mm}$ and $\varepsilon_{\text {mix }}=5 \cdot 0$. (c) Reconstruction based on the improved LBP. (d) Reconstruction by the new algorithm. The initial dielectric distribution was: $x=-10 \mathrm{~mm}, y=15 \mathrm{~mm}$, $r=10 \mathrm{~mm}$ and $\varepsilon_{\text {mix }}=5 \cdot 0$. (e) The square root of the object function as a function of the number of iterations for different initial dielectric distributions. 


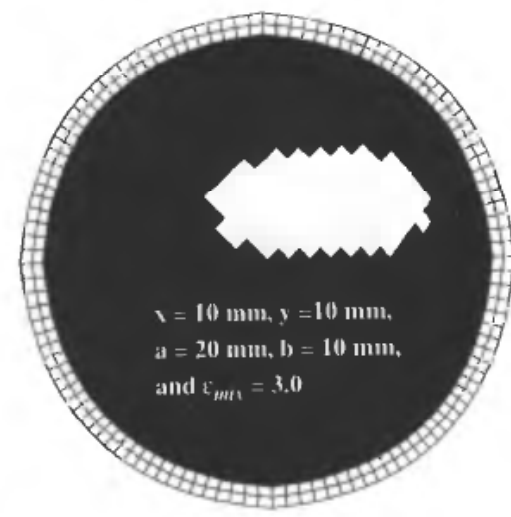

(a)

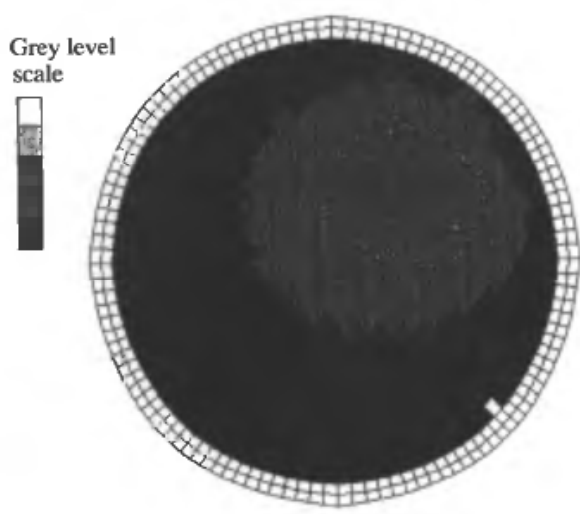

(c)

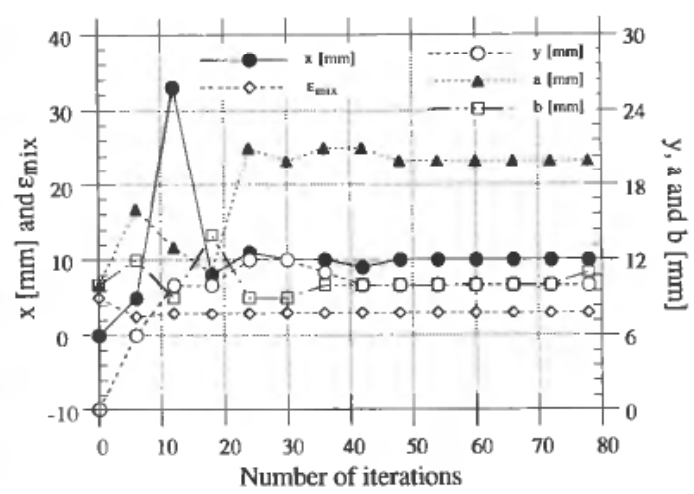

(b)

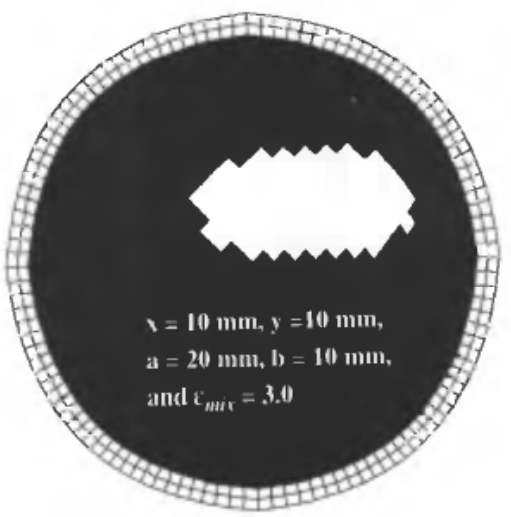

(d)

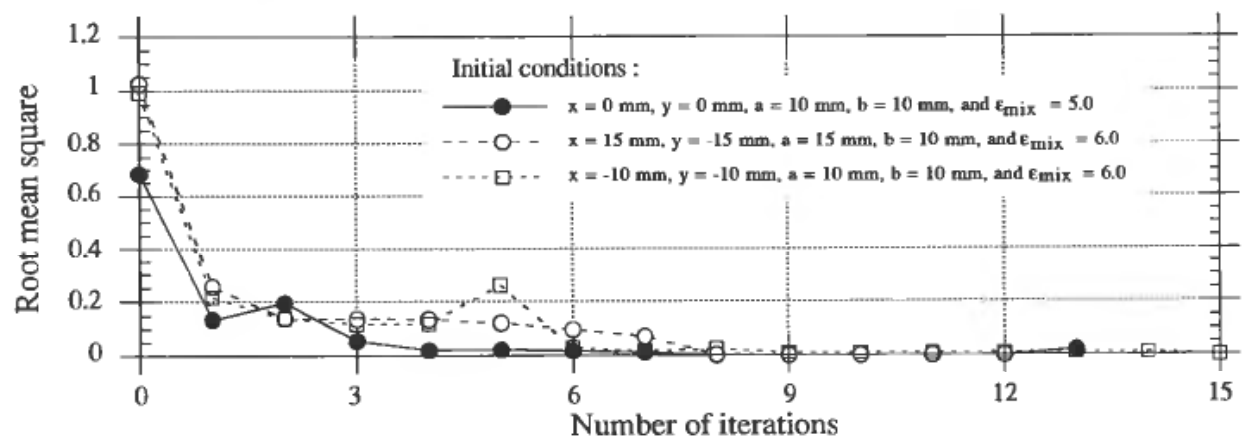

(e)

Figure 5. Reconstruction of an elliptical gas phase defined by $x=10 \mathrm{~mm}, y=10 \mathrm{~mm}, a=20 \mathrm{~mm}$, $b=10 \mathrm{~mm}$ and $\varepsilon_{\text {mix }}=3-0$. (a) The true oil/gas distribution. (b) $x, y, a, b$ and $\varepsilon_{\text {mix }}$ as a function of the number of iterations. The initial dielectric distribution was: $x=0 \mathrm{~mm}, y=0 \mathrm{~mm}$, $a=10 \mathrm{~mm}, b=10 \mathrm{~mm}$ and $\varepsilon_{\operatorname{mix}}=5 \cdot 0$. (c) Reconstruction based on the improved LBP. (d) Reconstruction by the new algorithm. The initial dielectric distribution was: $x=y$ $=0 \mathrm{~mm}, a=b=10 \mathrm{~mm}$ and $\varepsilon_{\text {mix }}=5 \cdot 0$. (e) The square root of the object function as a function of the number of iterations for different initial dielectric distributions. 


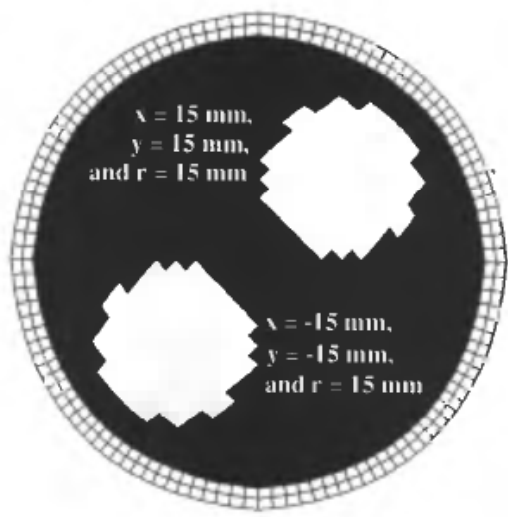

(a)

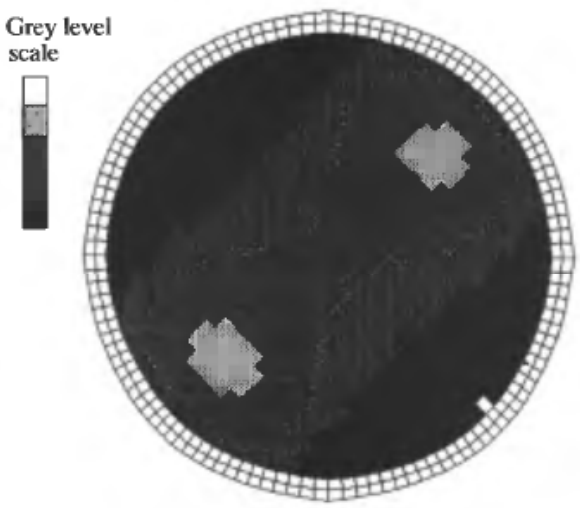

(c)

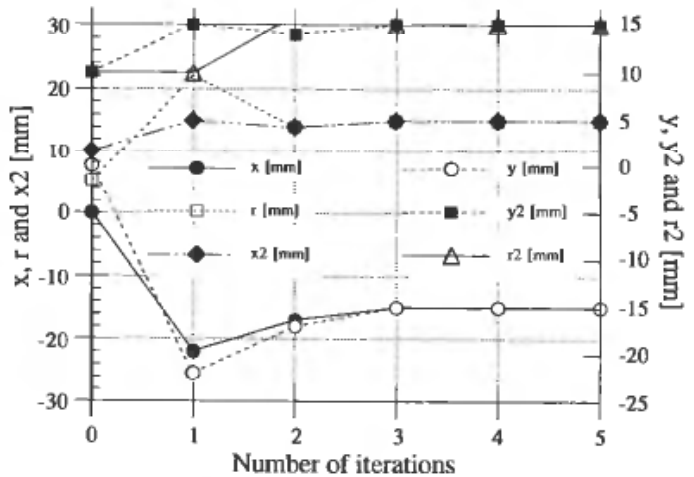

(b)

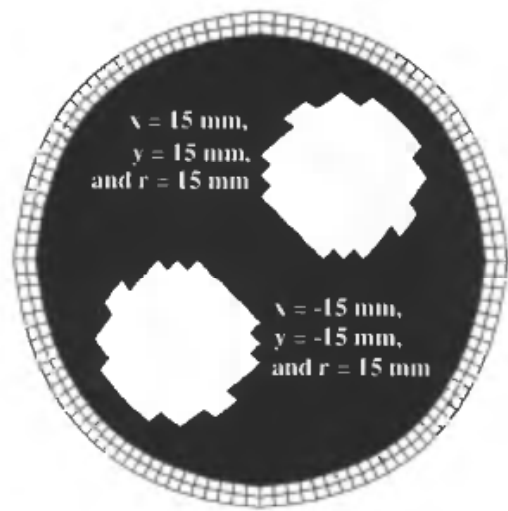

(d)

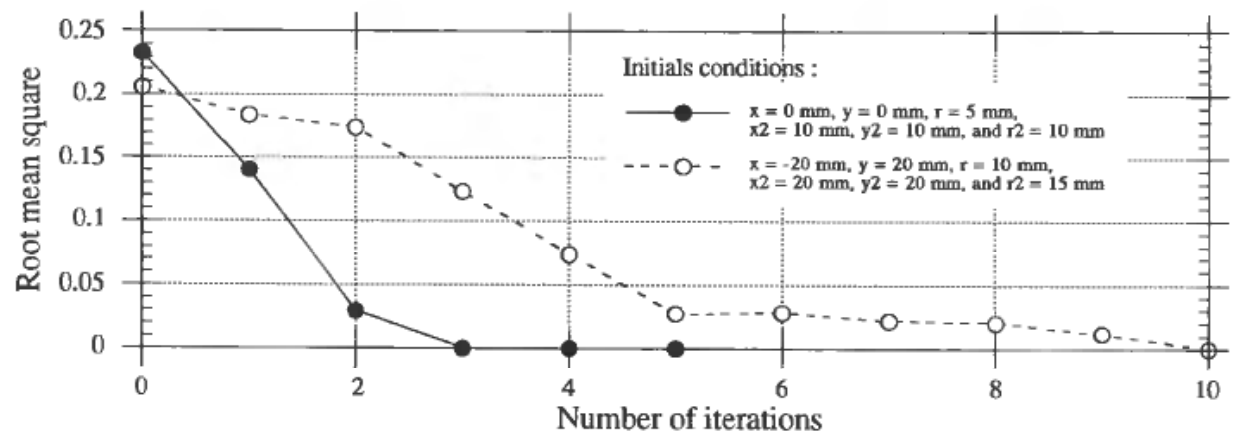

(e)

Figure 6. Reconstruction of two gas bubbles defined by $x=y=r=15 \mathrm{~mm}, x_{2}=y_{2}=-15 \mathrm{~mm}$ and $r_{2}=15 \mathrm{~mm}:(a)$ The true oil/gas distribution. $(b)$ The different parameters as a function of the number of iterations. The initial dielectric distribution was: $x=y=0 \mathrm{~mm}, r=5 \mathrm{~mm}$ and $x_{2}=y_{2}=r_{2}=10 \mathrm{~mm}$. (c) Reconstruction based on the improved LBP. (d) Reconstruction by the new algorithm. The initial dielectric distribution was $x=y=0 \mathrm{~mm}, r=5 \mathrm{~mm}$ and $x_{2}=y_{2}=r_{2}=10 \mathrm{~mm}$. (e) The square root of the object function as a function of the number of iterations for different initial dielectric distributions. 


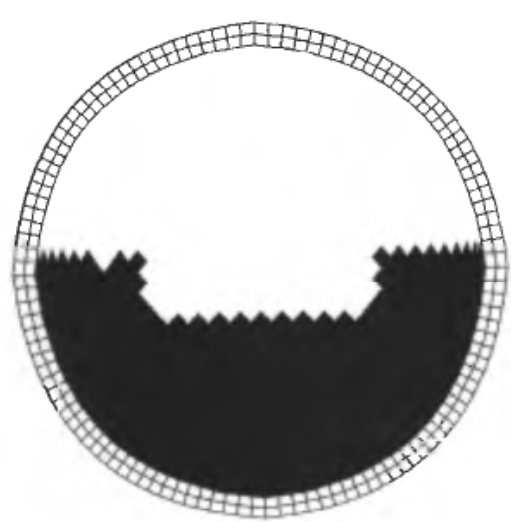

(a)

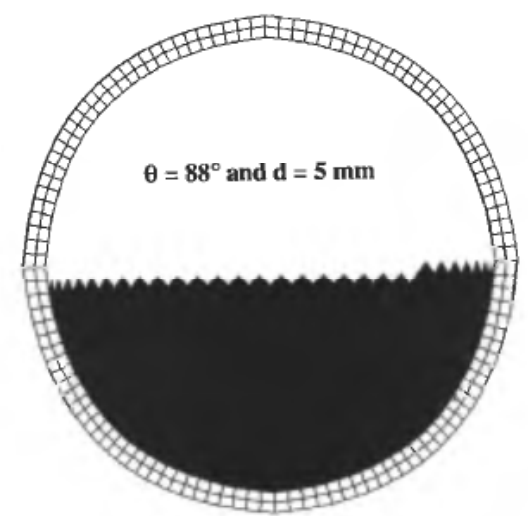

(b)

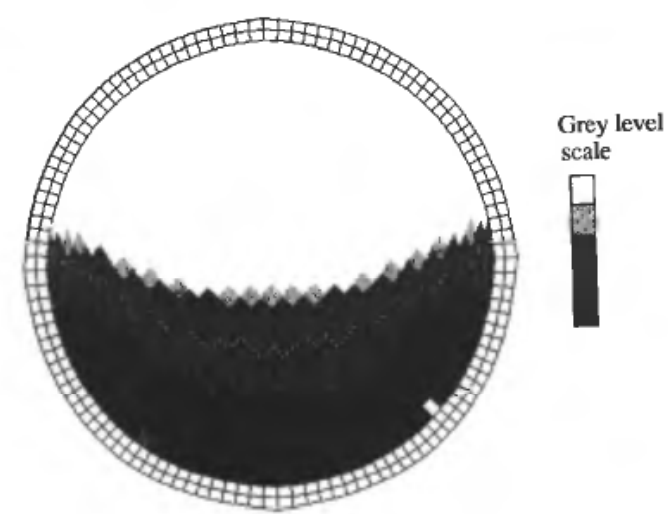

(c)

Figure 7. Reconstruction of a non-ideal stratified flow with both the improved LBP and the new reconstruction algorithm. The simple $\theta$ and $d$ parameterization shown in Fig. $3(c)$ is used. (a) The true oil/gas distribution. (b) Reconstruction by the new algorithm. (c) Reconstruction by the improved LBP.

different initial dielectric distributions. As can be seen from the figure, the number of iterations needed for the reconstruction is, in this case, rather independent of the initial dielectric distribution.

Figure 5 shows the reconstructions of an elliptically-shaped gas phase. In this case the dielectric constant for pure oil is 3.0; no gas is present in the oil continuous phase; see Fig. $5(a)$. The reconstruction based on the improved LBP indicates that a circular area of high gas concentration exists in the first quadrant. However, this is not the case. Again the reconstruction based on the new algorithm is successful.

Figure 6 shows the reconstruction of two gas bubbles situated in the first and third quadrants. The reconstruction based on the improved LBP indicates a high gas concentration in the first and third quadrants, but as before, it is hard to tell where the oil/gas interface is located. As can be seen from Fig. 6(d), the reconstruction using the new algorithm yields results identical to the true flow regimc.

Figure 7 shows a reconstruction of a non-ideal stratified flow using both the improved LBP and the new algorithm. In this case, the chosen parameterization ( $\theta$ and $d$ parameterization) cannot represent the flow regime shown in Fig. $7(a)$. However, as shown in Fig. 7 (b) the suggested $\theta$ and $d$ is a rather good estimate of the actual situation. The oil fraction in the reconstructed image is 0.458 , and the oil fraction in the simulated 
flow regime is equal to 0.454 . If this will be the case in general, the need for a more flexible parameterization is less important for many applications, however, a thorough investigation of this is still to be done.

\subsection{Results based on measured data}

Using a four-electrode sensor system (see Fig. 1) measurements on both annular and stratified flows have been conducted. The sensor construction parameters are: $41 \mathrm{~mm}$ inner pipe radius, $4 \mathrm{~mm}$ pipe wall made of perspex, screen radius equal to $60 \mathrm{~mm}$, and four electrodes mounted circumferentially around the pipe (the angle between two adjacent electrodes is $40^{\circ}$ ).

In Figs. 8 and 9, the reconstructions are based on measurements from the fourelectrode capacitance sensor system. The LBP based reconstruction of stratified flow (see Fig. 8(c)) clearly indicates a stratified flow regime, but it is hard to tell where the $\mathrm{oil} /$ gas interface is located as for the eight-electrode sensor system. As can be seen from Fig. $8(d)$, the reconstruction based on the new algorithm is quite good. Synthetic data (i.e., data generated with the capacitance simulator) generated using corresponding parameters (i.e., $\theta=90^{\circ}$ and $d=0 \mathrm{~mm}$ ) values was also reconstructed yielding the result: $\theta=92^{\circ}$ and $d=1 \mathrm{~mm}$. Hence, even by using synthetic data incorrect parameter values are obtained. This indicates that the error in the reconstruction is not only due to the inaccuracy in the mathematical model, the inaccuracy in the measurements, and/or the use of an improper finite element mesh, but also due to the lack of data supplied by a four electrode sensor system.

Figure 9 shows the reconstruction of annular flow. As can be seen from Fig. $9(c)$, the reconstruction based on the improved LBP clearly indicates annular flow, but again it is hard to locate the oil/gas interface. The reconstruction based on the new algorithm (see Fig. $9(d)$ ) is quite good. In both of the experimental cases (see Figs. 8 and 9), the reconstruction time was approximately 3.5 minute.

\subsection{Discussion}

The accuracy of the reconstruction based on the new algorithm is dependent on to what degree the parameterization (see Fig. 3) can represent the true (although unknown) flow regime, the accuracy of the mathematical model, and the accuracy in the measurements. Further, the information content in the capacitance data must uniquely define the flow regime, hence the object function must possess a well defined minimum. Figure 10 shows the square root of the object function as a function of $\theta$ and $d$. In this case, the true flow regime is a stratified flow given by $\theta=90^{\circ}$ and $d=10 \mathrm{~mm}$. As can be seen in Fig. 10, a well defined minimum exists at these parameters values.

Figure 10 is based on simulated 'measurements'. If the results had been based on physical measurements, the variance in the measurements and the inaccuracy in the mathematical model could have led to a minimum for the object function different from what is shown in Fig. 10. This problem can be solved by developing an accurate mathematical model of the physical process and by constructing high quality sensor electronics. Thus, the error due to inadequate mathematical description and experimental error may be reduced to an insignificant level. However, errors due to the inability of the parameterization to represent the true (although unknown) flow regime, as yet remains an unsolved problem. In this paper, flow regimes are represented by parameterizations containing between two and six parameters. Despite this simplicity, 


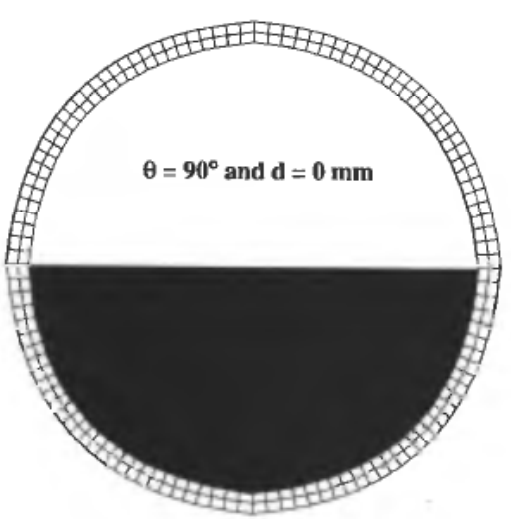

(a)

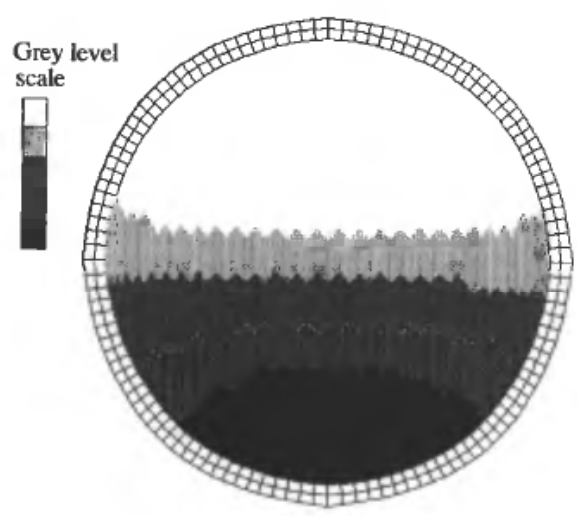

(c)

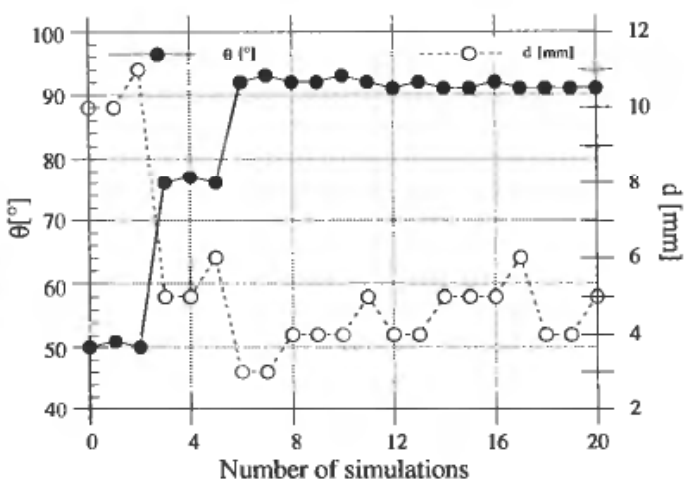

(b)

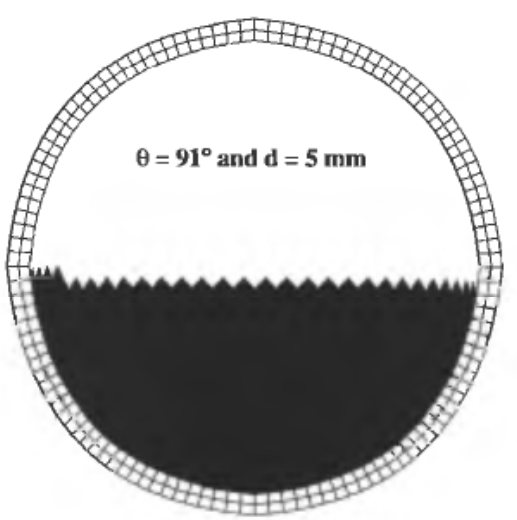

(d)

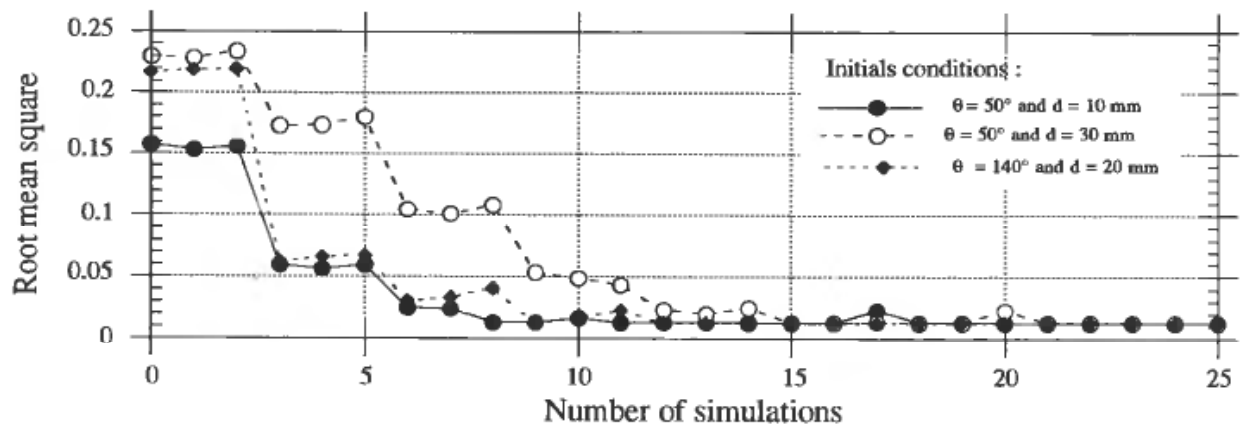

(e)

Figure 8. Reconstruction of stratified flow defined by $\theta=90^{\circ}$ and $d=0 \mathrm{~mm}$ : (a) The true oil/gas distribution. (b) $\theta$ and $d$ as a function of the number of simulations. The initial dielectric distribution was: $\theta=50^{\circ}$ and $d=10 \mathrm{~mm}$. (c) Reconstruction by the improved LBP. (d) Reconstruction using the new algorithm. The initial dielectric distribution was: $\theta$ $=50^{\circ} \mathrm{mm}$ and $d=10 \mathrm{~mm}$. (e) The square root of the object function as a function of the number of simulations for different initial dielectric distributions. 


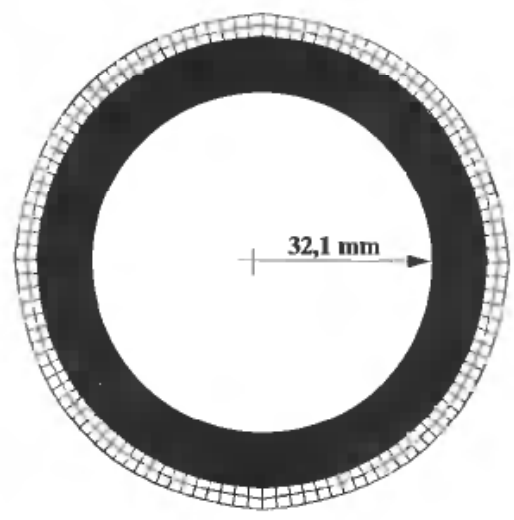

(a)

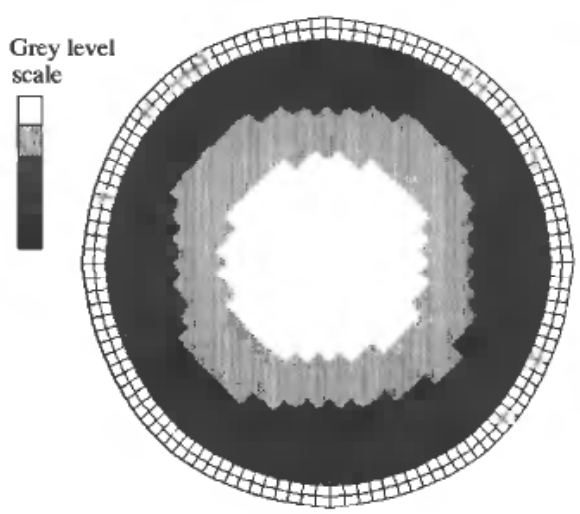

(c)

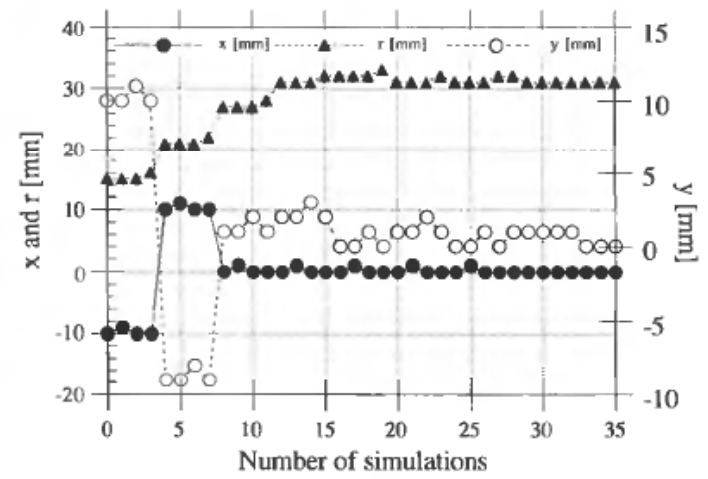

(b)

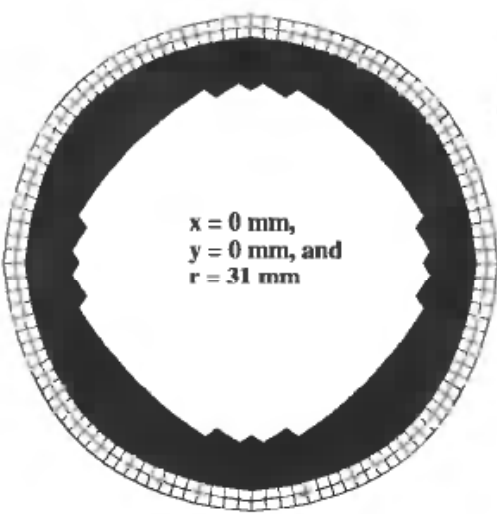

(d)

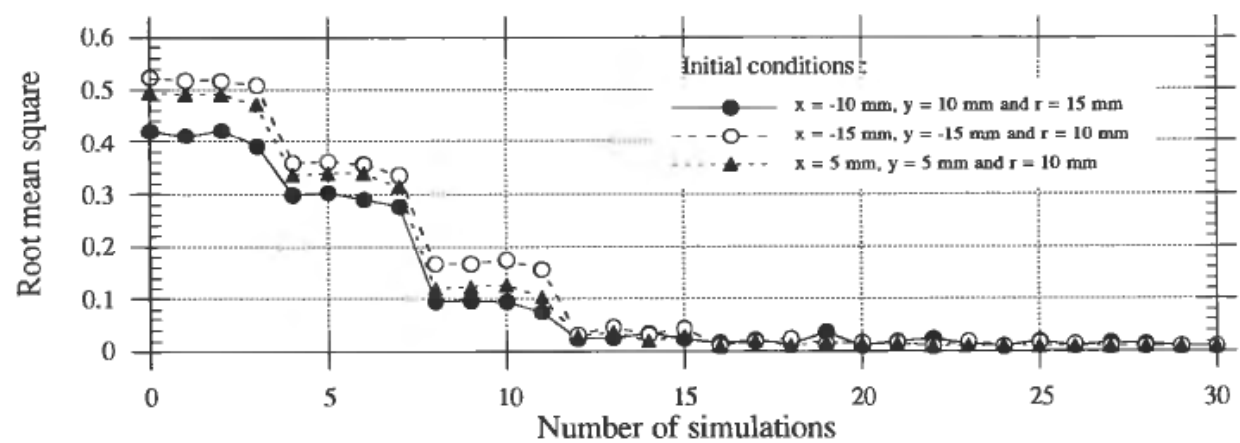

(e)

Figure 9. Reconstruction of annular flow defined by $x=y=0 \mathrm{~mm}$ and $r=32 \cdot 1 \mathrm{~mm}$. (a) The true oil/gas distribution. (b) $x, y$, and $r$ as a function of the number of simulations. The initial dielectric distribution was: $x=-10 \mathrm{~mm}, y=10 \mathrm{~mm}$ and $r=15 \mathrm{~mm}$. (c) Reconstruction by the improved LBP. (d) Reconstruction using the new algorithm. The initial dielectric distribution was: $x=-10 \mathrm{~mm}, y=10 \mathrm{~mm}$ and $r=15 \mathrm{~mm}$. $(e)$ The square root of the object function as a function of the number of simulations for different initial dielectric distributions. 


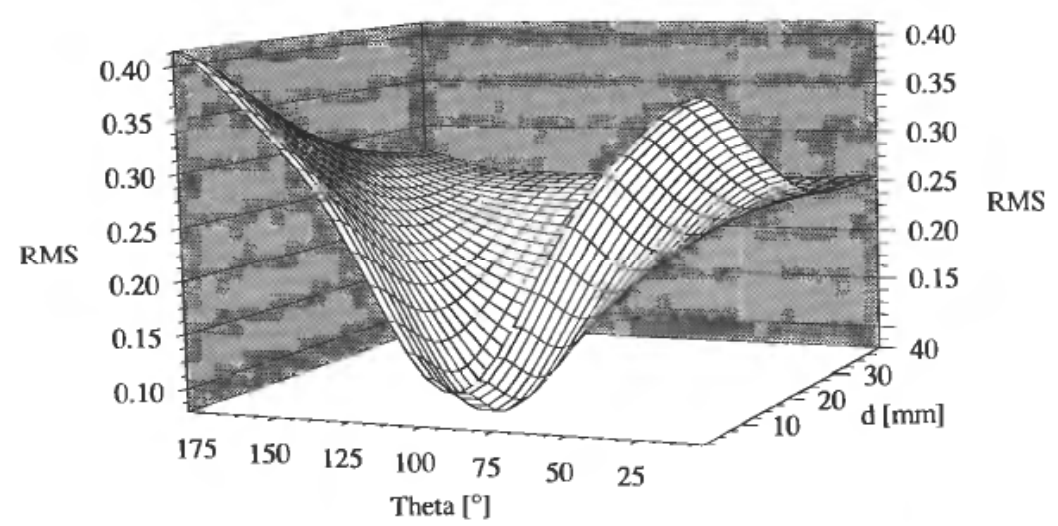

Figure 10. The square root of the object function calculated around the true flow regime, given by $\theta=90^{\circ}$ and $d=10 \mathrm{~mm}$ (an eight-electrode system).

the reconstruction based on these parameterizations may indeed be adequate for several applications.

The time used to reconstruct an image with the new algorithm is, at this stage, far too long to be used in an on-line application. For the simplest parameterization (the $\theta$ and $d$ parameterization) the reconstruction time used on an HP720 (a 17 MFLOPS RISC-based workstation) is approximately four minutes. The simulator is the most time consuming part of the reconstruction, hence, much can be gained by improving the speed of the capacitance simulator. This can be done by optimizing the code, implementing the code on a faster computer system, and/or calculating the capacitances on a coarser, but more appropriate finite element mesh. The optimal finite element mesh to be used can be obtained by an LBP based reconstruction. More flexible parameterization will also improve the usefulness of the method.

\section{Conclusions}

In this paper, a new method for interpreting data from process tomography systems has been proposed. An algorithm capable of reconstructing the flow pattern inside a pipe filled with oil and gas, using data from a capacitance tomography system has been developed. The method proves to be very promising, and represents a step towards quantitative capacitance tomography. The results showed in this paper are based on simple parameterization of the flow regimes, but for many applications this parameterization will, compared with the LBP method, give better quantitative information.

The new algorithm is based on an optimization algorithm and on a mathematical model of the sensor system. The present reconstruction time used is very long due to the fact that the simulator which calculates the capacitances for different flow regimes has to be executed several times during the reconstruction. However, this simulation time can be significantly reduced by optimizing both the capacitance model and the optimization algorithm, and/or by implementing the reconstruction algorithm on a faster computer system.

The proposed reconstruction algorithm has, in this work, been tested by using a capacitance based tomography system imaging oil/gas flow. However, in principle, the new reconstruction algorithm is indeed independent of both the sensor configuration and the flow media. 


\section{ACKNOWLEDGMENTS}

We are indebted to the Royal Norwegian Council for Scientific and Industrial Research for partially supporting this work. We would also like to thank Jarle Tollefsen, Dept. of Physics, University of Bergen, for providing 3D results which we could use to compare with our 2D capacitance model.

\section{REFERENCES}

AGA, M. (1991). Using the Levenberg-Marquardt Algorithm and the $B$-spline Representation to Determine the Characteristic Properties of the Porous Medium. MSc Thesis. University of Bergen, Norway.

Gill, P. E., Murray, W., and Wright, M. H. (1981). Practical Optimization (London, Academic Press).

Huang, S. M., Plaskowski, A. B., XIE, C. G. and BeCK, M. S. (1989). Tomographic imaging of two-component flow using capacitance sensors, J. Phys. E: Sci. Instrum., 22, 173-177.

IsAKSEN, Ø. (1989). Imaging Two-component Pipe Flow by Capacitance Sensors, MSc Thesis, University of Bergen, Norway.

ISAKSEN, Ø. and NORDTVEDT, J. E. (1992). Capacitance Tomography: Reconstruction Based on Optimization Theory, presented at the 1st European Concerted Action on Process Tomography (ECAPT) conference, 26-29 March, Manchester, UK. Tomographic Techniques for Process Design and Operation (Computational Mechanics Publication), 213-224.

Nordtvedt, J. E., Mesia, G., Yang, P. and Watson, A. T. (1991). Estimation of Capillary Pressure and Relative Permeability Functions From Centrifuge Experiments. Paper Society of Petroleum Engineers (SPE) 20805 accepted for publication in SPE Reservoir Engineering (SPERE).

Watson, A. T., Kerig, P. D., Richmond, P. C. and TAO, T. M. (1988). A Regression-Based Method for Estimating Relative Permeabilities From Displacement Experiments. SPERE (March 1988), 3.

XIE, C. G., Huang, S. M., Hoyle, B. S. and BeCK, M. S. (1991). Tomographic imaging of industrial equipment-development of system model and image reconstruction algorithm for capacitive tomography. Reviewed Proceedings of the 5th Conference on Sensors and Their Applications (Bristol: Adam Hilgar), 203-208.

Xie, C. G., Huang, S. M., Hoyle, B. S., Thorn, R., Lenn, C. and Beck, M. S. (1992). Electrical Capacitance Tomography for Flow Imaging-System model for development of reconstruction algorithms and design of primary sensors. IEE Proceedings $\mathrm{G}, 139,89-98$. 